\title{
An Equivalent Boundary Model for Effects of Adjacent Spans on Wind Reliability of Wood Utility Poles in Overhead Distribution Lines
}

\author{
Yousef Mohammadi Darestani ${ }^{*}$, Abdollah Shafieezadeh ${ }^{\dagger}$, and Reginald DesRoches ${ }^{\ddagger}$
}

\begin{abstract}
Overhead distribution lines in the United States are primarily supported by wood poles. These utility poles are vulnerable to high wind loads from hurricanes which has resulted in a large number of power outages during past hurricanes, especially in coastal areas. Risk management of distribution systems requires development of distribution line models to reliably assess their performance under hurricanes. However, modeling the entire distribution line is not practically feasible as they are very long and consist of a large number of spans. The present study proposes analytical models to capture boundary effects of adjacent spans on the wind response of the pole of interest via equivalent springs and wind-induced forces. The stiffness of conductors (cables) is derived through a simplified solution that considers the shape of the conductors under lateral static gust wind and gravity loadings; this model is shown to be very accurate when compared to the exact analytical as well as Finite Element solutions. Deterministic and probabilistic studies are performed to assess the impact of boundary conditions on the performance of the poles; results indicate that pole boundaries may have a considerable impact on the estimates of failure probabilities especially when the extent of difference in the properties of adjacent spans is not negligible. Specifically, neglecting boundary effects from neighboring spans may result in underestimation of failure probabilities of stronger poles and overestimation of failure probabilities of weaker poles as load sharing effects among the poles are not considered. The proposed boundary model enables more reliable hurricane risk assessment of power distribution systems to effectively manage the risk of outages.
\end{abstract}

Keywords: Wood utility poles; fragility analysis; hurricane hazard; boundary effects; aging and deterioration

\section{INTRODUCTION}

Electricity supports various activities in a society and any disruption in its constant flow may result in large economic loss. The infrastructure supporting electricity faces risk of damage from various natural hazards such as hurricanes, especially in coastal areas. Hurricanes are responsible for the majority of power outages in the United States. For instance, in 1992, hurricane Andrew caused 1.4 million customers to lose their power in Florida (Larsen et al., 1996). Also, Hurricane Sandy in 2012 caused 8.5 million customers to lose power leading to over $\$ 50$ billion in economic loss (Blake et al., 2013). The electrical power system consists of three major units including generation, transmission and distribution; among these, distribution lines suffered extensive damage during past hurricanes (Davidson et al., 2003). In the United States, the majority of utility poles are made

\footnotetext{
* Department of Civil, Environmental and Geodetic Engineering, Ohio State University, Columbus, US

${ }^{\dagger}$ Department of Civil, Environmental and Geodetic Engineering, Ohio State University, Columbus, US;

Corresponding author: Shafieezadeh.1@osu.edu;

‡ School of Civil and Environmental Engineering, Georgia Institute of Technology, Atlanta, US
} 
of wood. Although poles made of wood are widely available and less costly, they sustain considerable damage especially under strong wind hazards. Past hurricanes caused severe damage to distribution lines. In 1989, Hurricane Hugo destroyed over 15,000 poles, over 6,000 transformers and about 700 miles of conductors (cables) (Johnson, 2004). In 2005, over 12,000 Florida Power and Light (FPL) utility poles were failed during Hurricane Wilma and Hurricane Katrina (Brown, 2006).

The high vulnerability of distribution lines during past hurricanes highlights the need for risk assessment methods in order to manage the risk of outages induced by failure of distribution systems. Such risk assessment methods require reliable performance assessment of distribution lines using models that can capture their actual behavior during extreme wind events. A distribution line can be as long as several miles with hundreds of poles. Therefore, numerical modeling of the entire system using methods such as Finite Element techniques to analyze the performance of distribution lines under wind loadings is not practical. A solution to this problem is to assume that all poles and all spans in the distribution line are the same; therefore, effects of neighboring spans can be neglected. Shafieezadeh et al., (2014) developed fragility models of aged Southern Pine wood poles for the US region. In their analysis, potential effects of neighboring spans were not considered and only the pole of interest was modeled. This simplifying assumption was used in a number of studies on the wind performance of poles and distribution lines in the US and other areas (Ryan et al., 2014; Salman and Li, 2015; and Salman et al., 2015). However, this simplifying assumption may not produce accurate results for actual distribution lines. In order to have a reliable electric distribution system, utility poles are inspected every few years (Onyewuchi et al., 2015). Based on the inspection results, some of the decayed and damaged poles are strengthened or replaced by new poles. Therefore, in an actual distribution line, it is very likely that the age distribution of the poles and, in general, their condition state is not uniform. Depending on the extent of difference between properties of adjacent poles and spans, boundary conditions may have a significant impact on the performance of the poles and spans of interest. It should be noted that if there is any considerable change in the stiffness and wind-induced forces in the neighboring spans, boundary effects must be considered. Factors that can result in the variation in the stiffness of poles is not limited to the age of the poles; geometry of the poles, span length, number and diameter of conductors, and wind speed and direction are among significant variables that can also cause variation in the stiffness and the wind-induced forces in adjacent spans.

The present study proposes a model based on equivalent static boundary conditions to capture impacts of neighboring spans for wind performance assessment of distribution lines. The boundary model consists of equivalent springs and forces corresponding to the stiffness of the poles and lines and wind-induced forces over the length of the distribution line. To obtain the equivalent boundary conditions, the exact model for three-dimensional deformed shape of conductors is derived considering general boundary conditions when conductors are subjected to static gust wind and gravity loadings. Furthermore, a simple parabolic approximate model is derived for the deformed shape of conductors. These two models are validated against Finite Element solutions for conductors. Using the approximate model, a stiffness matrix and a force vector are assembled to account for the wind response of distribution line segments considering the variation of stiffness in the spans and corresponding wind-induced forces over the length of distribution lines. Based on these models, a static boundary condition for the effects of adjacent spans is defined through an equivalent stiffness and equivalent force. Using these models, a series of deterministic and probabilistic studies are conducted on a set of wood utility poles of different ages in a long overhead distribution line. The rest of this paper is outlined as follows: section 2 
presents the derivation of the analytical boundary condition model for utility poles. Section 3 explains the process for determination of failure probabilities given the age of the poles and boundary effects. Results of numerical studies and conclusions of this research are presented in section 4 and 5, respectively.

\section{AN ANALYTICAL BOUNDARY CONDITION MODEL FOR UTILITY POLES 2.1. Model Description}

Since distribution lines are very long and consist of a large number of spans, modeling the entire system is not practically feasible for performance assessment of utility poles. As shown in Figure 1.a, poles in a distribution line are connected through conductors (In the United States, cables that are used in electric power lines to carry electricity are often called "conductors"). Therefore, the response or performance of any segment of a distribution line is impacted by the rest of the system. This study proposes equivalent boundary conditions to capture the impact of the neighboring spans to assess the wind response of a single utility pole, represented by $P_{i}$ in Figure 1.a. However, the methodology can be utilized to define the boundary conditions for any segment of a distribution line. As shown in Figure 1.b, under static analysis, the impact of the rest of a distribution line on the pole of interest can be defined through the equivalent boundary conditions which are composed of an equivalent spring representing the stiffness of the adjacent spans and a wind-induced equivalent force to account for the forces applied from neighboring spans to the pole of interest. In Figure 1.a, pole $P_{i}$ is considered as the pole of interest. In this study, it is assumed that the direction of wind (transverse-y direction) is perpendicular to the distribution line (longitudinal- $\mathrm{X}$ direction). This assumption represents most probably the worst case scenario for the impact of wind on overhead distribution lines; this is due to the fact that the projected wind area for conductors is maximum when the direction of the wind is perpendicular to the direction of the distribution line. In order to define boundary conditions, the stiffness and equivalent wind-induced forces must be obtained for poles, cross arms and conductors in each span of the distribution line. The stiffness and equivalent force of poles can be calculated when the modulus of elasticity, moment of inertia, height, and profile of the wind for each of the utility poles are known. Since the impact of cross arms on the response of the poles when subjected to transverse winds is negligible, stiffness and equivalent force of these components are not considered in this study. The models for the contribution of poles and conductors to the stiffness and equivalent force in the boundary model are derived via the application of equilibrium and compatibility equations. This process is outlined in the subsequent sections. 


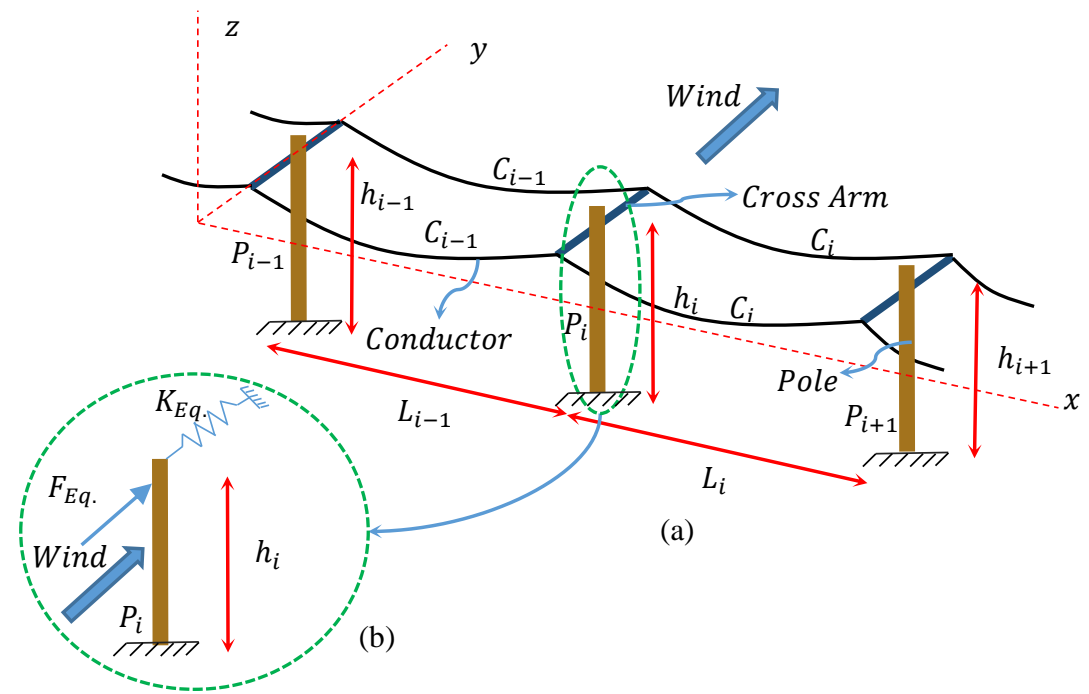

Figure 1. Schematic of a typical distribution line: (a) configuration and constitutive components of the distribution line and (b) the pole of interest with equivalent boundary conditions.

\subsection{Equivalent Stiffness and Wind Force of Poles}

As discussed in the previous section, in order to obtain the equivalent boundary conditions, the stiffness and equivalent force for each component of the distribution line must be derived. For utility poles, a flexibility method can be used to calculate their stiffness. As shown in Figure 2.a, this method entails applying a unit force to the point at the intersection of poles and cross arms (point A) and deriving the displacement of this point as follows.

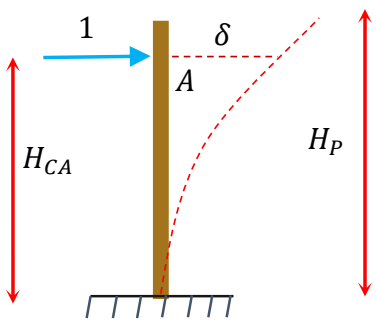

a)

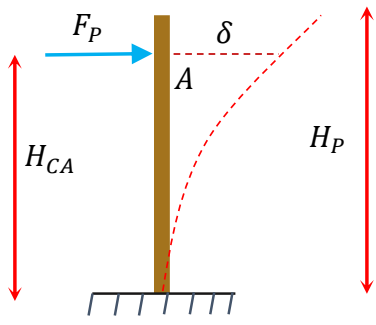

b)

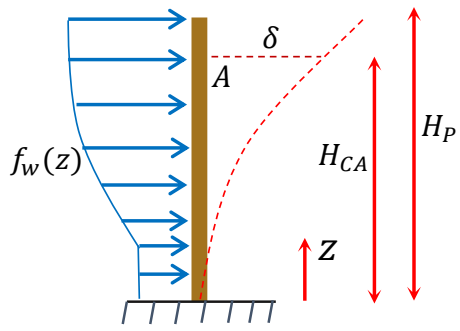

c)

Figure 2. Obtaining stiffness and equivalent force of the poles a) Flexibility method to obtain Stiffness b) The equivalent force applied to point $A$ c) The wind force applied to the pole.

$$
\delta=\int_{0}^{H_{C A}} \frac{z^{2}}{E_{w} \cdot I(z)} d z
$$

where $H_{C A}$ is the height of the cross arm from ground line, $z$ is the height from ground line, $E_{w}$ is the modulus of elasticity of the pole, and $I(z)$ is the moment of inertia of the pole at height $z$. The inverse of the displacement of point $A$ due to a unit load is defined as the stiffness of the structure.

$$
K_{p}=1 / \delta
$$

In order to derive the equivalent force of the poles at point $A$, the power wind profile as suggested by ASCE07 (2010) is utilized. The wind force per unit length for a non-building structure can be determined from:

$$
f_{w}=q_{z} G C_{f} D
$$


where $q_{z}$ is the velocity pressure at height $z$ on the pole, $G$ is the gust-effect factor, $C_{f}$ is the force coefficient, and $D$ is the diameter perpendicular to the wind direction. The wind pressure is obtained from:

$$
q_{z}=0.613 K_{z} K_{d} K_{z t} V^{2} I
$$

where $K_{z}$ is the velocity pressure exposure coefficient, $K_{d}$ is the wind directionality factor, $K_{z t}$ is the topographic factor, $V$ is the 3 -second gust wind speeds at $10 \mathrm{~m}$ above the ground, and $I$ is the importance factor. In order to make the model independent of topographic conditions, this study assumes $K_{z t}$ to be one. Importance factor for utility poles is also taken as one. According to ASCE07 (2010), $K_{z}$ is a function of the height from the ground line and exposure category and can be obtained from:

$$
K_{z}=2.01\left(\frac{\max (4.75, z)}{z_{g}}\right)^{2 / \alpha}
$$

It is assumed that the distribution line is located in an open terrain area; therefore, the exposure category according to ASCE07 (2010) is C, and $\alpha$ and $z_{g}$ are 9.5 and $274.32 \mathrm{~m}$, respectively. The wind directionality factor, $K_{d}$, is taken as 0.95 per ASCE07 (2010) recommendation. The gusteffect factor, $G$, accounts for the effects of the dynamic nature of wind forces on the pole. When the structure is rigid, $G$ can be taken as 0.85 (ASCE07, 2010). The force coefficient, $C_{f}$, depends on the surface roughness, the height to diameter ratio and velocity pressure, $q_{z}$. This parameter can be determined using the linear interpolation for $h / D$ values in Table 1 . Table 1 is adopted from ASCE07 (2010) assuming the surface of wood poles is rough. For $h / D$ values larger than $25, h / D$ is assumed to be equal to 25 .

Table 1. The Force Coefficient, $\mathrm{C}_{\mathrm{f}}$ from ASCE7 (2010)

\begin{tabular}{lccc}
\hline & \multicolumn{3}{c}{$h / D$} \\
\cline { 2 - 4 } & 1 & 7 & 25 \\
\hline$D \sqrt{q_{z}}>5.3$ & 0.7 & 0.8 & 0.9 \\
\hline$D \sqrt{q_{z}} \leq 5.3$ & 0.7 & 0.8 & 1.2 \\
\hline
\end{tabular}

In order to find the equivalent force at point $A$, the displacement due to equivalent force at that point should be equal to the displacement due to wind pressure over the height of the pole. The displacement at the point $A$ due to the equivalent force is:

$$
\delta=\frac{F_{p}}{K_{p}}
$$

where $F_{p}$ is the equivalent wind force on the pole at point $A$ as shown in Figure 2.b. The displacement due to wind force (Figure 2.c) is obtained using the principal of virtual work.

$$
\delta=\int_{0}^{H_{C A}} \frac{M(z)\left(H_{C A}-z\right)}{E_{w} I(z)} d z
$$

where $M(z)$ is the moment at height $z$ :

$$
M(z)=\int_{z}^{H_{p}} f_{w}(\mu)(\mu-z) d \mu
$$

In this equation, $f_{w}(\mu)$ is the wind force per unit length at height $\mu$ and $H_{P}$ is the height of the pole from the ground line. The equivalent wind force on the pole can be determined from Eq. (6) and (7): 


$$
F_{p}=\frac{\int_{0}^{H_{C A}} \frac{\left[\int_{Z}^{H_{p}} f_{w}(\mu)(\mu-z) d \mu\right]\left(H_{C A}-Z\right)}{I(z)} d z}{\int_{0}^{H_{C A}} \frac{Z^{2} d z}{I(z)}}
$$

\subsection{Equivalent Stiffness and Wind Force of Conductors}

\subsubsection{Exact deformed shape of conductors under gravity and lateral gust wind loadings}

As mentioned earlier, winds in the transverse direction (parallel to y-axis) induce the largest stress in utility poles in distribution lines. The stiffness of conductors in the y-direction depends on their deformed shape under static gust wind and gravity forces as shown in Figure 3.a. The equilibrium equations for the free body diagram of a cable element (Figure 3.b) in Cartesian coordinates yield

$$
\begin{aligned}
& \frac{d}{d s}\left(\frac{T d x}{d s}\right) \Delta s=0 \\
& \frac{d}{d s}\left(\frac{T d y}{d s}\right) \Delta s+f_{w} \Delta s=0 \\
& \frac{d}{d s}\left(\frac{T d z}{d s}\right) \Delta s-m g \Delta s=0
\end{aligned}
$$

where $T$ is the tension in the cable, $m g$ is the weight per unit length of the cable and $f_{w}$ is the static gust wind force per unit length of the cable in y-direction. Also, $d s$ can be represented as:

$$
d s=\sqrt{(d x)^{2}+(d y)^{2}+(d z)^{2}}
$$

Integrating Eq. (10) results in

$$
T \frac{d x}{d s}=H
$$

where $H$ is the horizontal component of the tensile force in the conductor. Replacing Eq. (14) into Eqs. (11) and (12) yields

$$
\begin{aligned}
& H \frac{d^{2} y}{d x^{2}}=-f_{w} \frac{d s}{d x} \\
& H \frac{d^{2} z}{d x^{2}}=m g \frac{d s}{d x}
\end{aligned}
$$

Replacing Eq. (13) into Eqs. (15) and (16) leads to

$$
H \frac{d^{2} y}{d x^{2}}=-f_{w} \sqrt{1+\left(\frac{d y}{d x}\right)^{2}+\left(\frac{d z}{d x}\right)^{2}}
$$

and

$$
H \frac{d^{2} z}{d x^{2}}=m g \sqrt{1+\left(\frac{d y}{d x}\right)^{2}+\left(\frac{d z}{d x}\right)^{2}}
$$

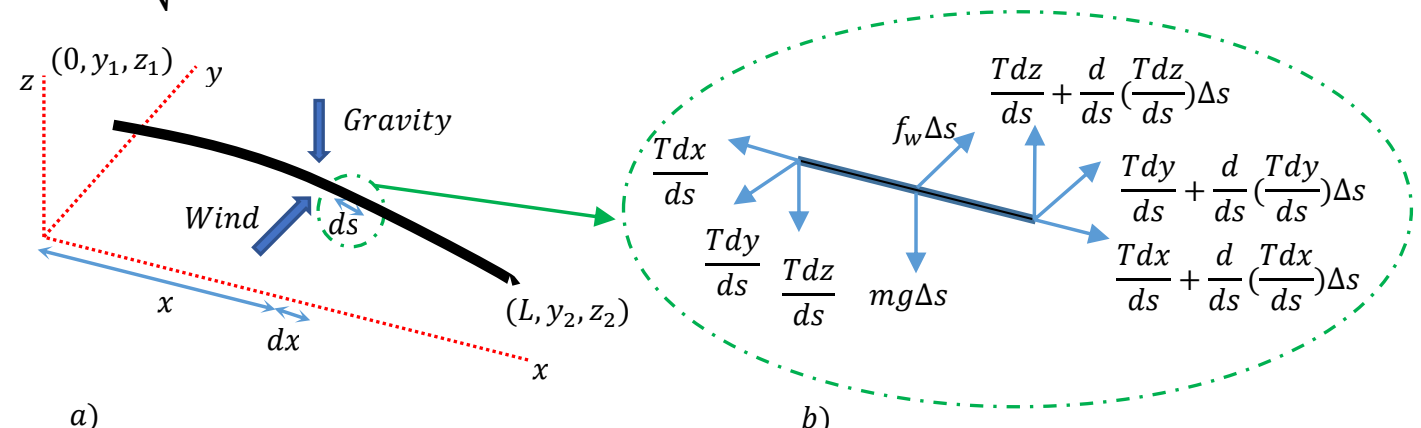


Figure 3. A typical conductor a) under static gust wind and gravity loading b) free body diagram of a cable element.

Equations (17) and (18) need to be solved simultaneously because they are coupled. These equations can be decoupled by transforming the coordinate system from $(x, y, z)$ to $(x, \xi, \eta)$ as shown in Figure 4. The new coordinate system is set such that $\xi$ is parallel to the equivalent applied force from static gust wind and gravity wind loadings.

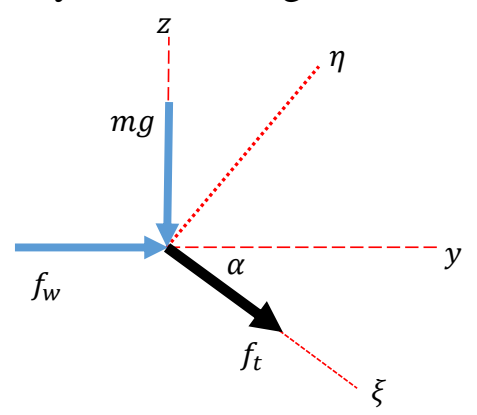

Figure 4. Change of coordinates for finding the exact shape of the cable

Equations (17) and (18) in the new coordinate system are:

$$
H \frac{d^{2} \xi}{d x^{2}}=-f_{t} \sqrt{1+\left(\frac{d \xi}{d x}\right)^{2}+\left(\frac{d \eta}{d x}\right)^{2}}
$$

and

$$
H \frac{d^{2} \eta}{d x^{2}}=0
$$

The equivalent force, $f_{t}$, and the angle of $\xi$ with respect to $\mathrm{y}, \alpha$, in Figure 4 are:

$$
f_{t}=\sqrt{(m g)^{2}+\left(f_{w}\right)^{2}}
$$

and

$$
\alpha=\tan ^{-1}\left(\frac{m g}{f_{w}}\right)
$$

The coordinates for the two ends of the cable in Figure 3.a are $\left(0, y_{1}, z_{1}\right)$ and $\left(L, y_{2}, z_{2}\right)$. Using the following transformation, the boundary conditions are transformed to the new coordinate system.

$$
\left\{\begin{array}{l}
\xi \\
\eta
\end{array}\right\}=\left(\begin{array}{cc}
\cos (\alpha) & -\sin (\alpha) \\
\sin (\alpha) & \cos (\alpha)
\end{array}\right)\left\{\begin{array}{l}
y \\
z
\end{array}\right\}
$$

Applying these boundary conditions to Eqs. (12) and (13), $\xi$ and $\eta$ are found as

$$
\begin{aligned}
& \eta=\left[\left(\frac{y_{2}-y_{1}}{L}\right) \sin (\alpha)+\left(\frac{z_{2}-z_{1}}{L}\right) \cos (\alpha)\right] x+y_{1} \sin (\alpha)+z_{1} \cos (\alpha) \\
& \xi=a-\frac{H}{f_{t}} \sqrt{1+\left[\left(\frac{y_{2}-y_{1}}{L}\right) \sin (\alpha)+\left(\frac{z_{2}-z_{1}}{L}\right) \cos (\alpha)\right]^{2}} \cosh \left(-\frac{f_{t}}{H} x+d\right)
\end{aligned}
$$

where $d$ and $a$ are calculated by replacing the two end boundary conditions for $\xi$ into Eq. (25) and solving the resulting coupled system of nonlinear equations. Finally, the exact shape of the conductor under static gust wind and gravity forces in $(x, y, z)$ coordinate system can be obtained using:

$$
\left\{\begin{array}{l}
y \\
z
\end{array}\right\}=\left(\begin{array}{cc}
\cos (\alpha) & \sin (\alpha) \\
-\sin (\alpha) & \cos (\alpha)
\end{array}\right)\left\{\begin{array}{l}
\xi \\
\eta
\end{array}\right\}
$$


It should be noted that the produced models for the shape of conductors do not account for possible vibrations under dynamic wind loadings.

\subsubsection{Approximate deformed shape of conductors under lateral static gust wind and gravity loadings}

In order to obtain the equivalent stiffness of conductors, an approximate solution for their deformed shape is proposed. The accuracy of this model is assessed later in the numerical study section. If the sag in the conductor is small compared to the length of the conductor, $\frac{d y}{d x}$ and $\frac{d z}{d x}$ are negligible and therefore can be neglected in Eqs. (17) and (18). This assumption may not hold for some extreme cases. For example, elevated temperatures may result in thermal expansion of conductors and increase the sag. In such cases, the level of approximation in the derived solutions for the shape of conductors may increase. However, it should be noted that conductors are designed to experience a relatively small sag in order to meet the minimum clearance limits. Therefore, in this study it is assumed that the sag in the conductors is small compared to their length. Consequently, Eqs. (17) and (18) will be simplified to

$$
H \frac{d^{2} y}{d x^{2}}=-f_{w}
$$

and

$$
H \frac{d^{2} z}{d x^{2}}=m g
$$

Applying boundary conditions to these equations results in

$$
y=\frac{-f_{w}}{2 H} x^{2}+\left(\frac{f_{w} L}{2 H}-\frac{y_{1}-y_{2}}{L}\right) x+y_{1}
$$

and

$$
z=\frac{m g}{2 H} x^{2}-\left(\frac{m g}{2 H} L+\frac{z_{1}-z_{2}}{L}\right) x+z_{1}
$$

The equilibrium equation in y-direction at the point of interest yields

$$
-\left.T \frac{d y}{d x}\right|_{x=0}+K_{p} y_{1}-F_{p}=0
$$

where $T$ is the tensile force in the conductor, $K_{p}$ is the stiffness of the pole from Eq. (2) and $F_{p}$ is the equivalent force of the pole calculated using Eq. (9). Equation (29) provides a good approximation for the deformed shape of conductors under gust wind and gravity forces in the transverse direction when the sag is small. Using this equation, $\left.T \frac{d y}{d x}\right|_{x=0}$ in Eq. (31) can be rewritten as:

$$
\left.T \frac{d y}{d x}\right|_{x=0}=\frac{f_{w} L}{2}-\frac{H}{L}\left(y_{1}-y_{2}\right)
$$

Subsequently, the equivalent force, $F_{c}$, and the equivalent stiffness, $K_{c}$, of the conductor can be found as:

$$
F_{c}=\frac{f_{w} L}{2} ; K_{c}=\frac{H}{L}
$$

\subsection{Equivalent Stiffness and Wind Force of Distribution Lines}

After the stiffness and equivalent force for each component of the distribution line is obtained, a stiffness matrix and a force vector can be assembled in order to find the boundary conditions for 
the pole of interest. Let's assume there are $m$ lines of conductors and $n_{L}$ and $n_{R}$ spans at the left and right side of the pole of interest, respectively. Figure 5 provides a representation of poles and conductors at the right side of the pole of interest with equivalent springs and equivalent forces. A similar model can be developed for the distribution line at the left side of the pole of interest. For the system shown in Figure 5, the stiffness matrix and the force vector are

$$
\widetilde{K}_{R}=\left[\begin{array}{ccc}
\widetilde{K}_{U L} & \cdots & \widetilde{0} \\
\vdots & \ddots & \vdots \\
\tilde{0} & \cdots & \widetilde{K}_{B R}
\end{array}\right]
$$

where $\widetilde{K}_{U L}$ and $\widetilde{K}_{B R}$ are

$$
\widetilde{K}_{U L}=\left[\begin{array}{ccc}
K_{P, n_{R}+i}+m\left(K_{C, n_{R}+i}+K_{C, n_{R}+i-1}\right) & -m\left(K_{C, n_{R}+i}+K_{C, n_{R}+i-1}\right) & 0 \\
-m\left(K_{C, n_{R}+i}+K_{C, n_{R}+i-1}\right) & K_{P, n_{R}+i-1}+m\left(K_{C, n_{R}+i-1}+K_{C, n_{R}+i-2}\right) & -m K_{C, n_{R}+i-2}
\end{array}\right]
$$

and

$$
\widetilde{K}_{B R}=\left[\begin{array}{ccc}
-m K_{c, i+1} & K_{P}+m\left(K_{c, i}+K_{c, i+1}\right) & -m K_{c, i} \\
0 & -m K_{c, i} & m K_{c, i}
\end{array}\right]
$$

and

$$
\begin{aligned}
& \vec{F}_{R}=\left[F_{n_{R}+i-1}, \ldots F_{i+1}, F_{i}\right]^{T}= \\
& \quad\left[m\left(F_{c, n_{R}+i-1}+F_{c, n_{R}+i-2}\right)+F_{p, n_{R}+i-1}, m\left(F_{c, n_{R}+i-2}+F_{c, n_{R}+i-3}\right)+F_{p, n_{R}+i-2}, \ldots, m\left(F_{c, i}+F_{c, i+1}\right)+F_{p, i+1}, m F_{c, i}\right]^{T}
\end{aligned}
$$

The displacement vector is defined as

$$
\vec{U}=\left[u_{n_{R}+i-1}, u_{n_{R}+i-2}, \ldots, u_{i+1}, u_{i}\right]^{T}
$$

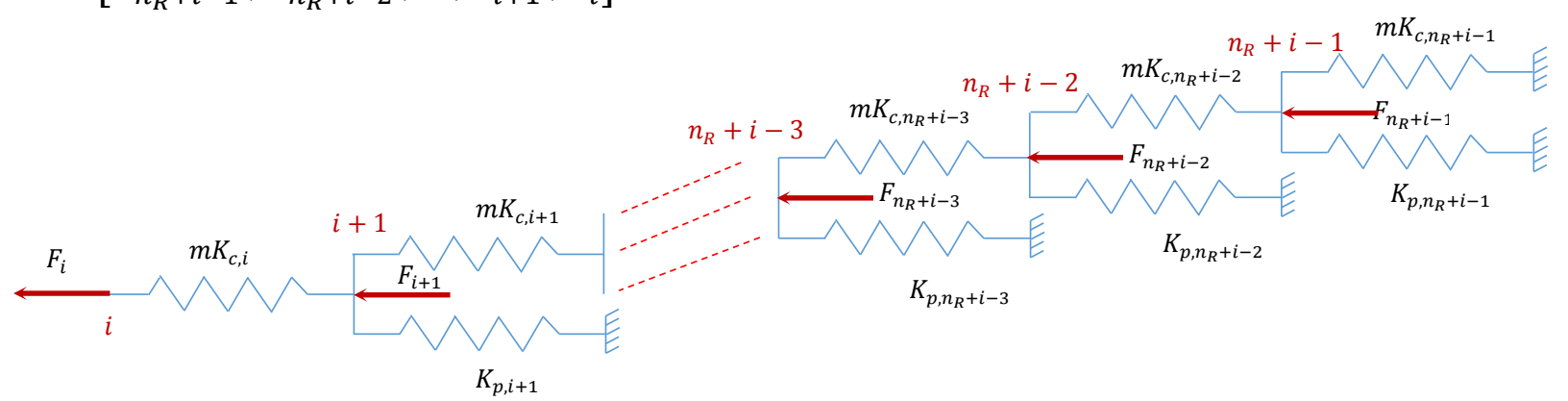

Figure 5. Representation of poles and conductors with equivalent springs and corresponding forces for the distribution line at the right side of the pole of interest.

Superposition is used to obtain the equivalent force at the boundary. In order to use superposition, node $i$ (the boundary node) is fixed (Figure 6.a) and the reaction force, $R_{e}$, is derived using

$$
\vec{R}_{e}+\vec{F}_{R}=\widetilde{K}_{R} \cdot \vec{U} ; \vec{U}=\left[u_{n_{R}+i-1}, u_{n_{R}+i-2}, \ldots, u_{i+1}, 0\right]^{T}
$$

where $\vec{R}_{e}$ is defined as

$$
\vec{R}_{e}=\left[\begin{array}{lllll}
0 & 0 & \ldots & 0 & -R_{e}
\end{array}\right]^{T}
$$

After $R_{e}$ is obtained, node $i$ is released and a force equal to $-R_{e}$ is applied to node $i$ (Figure 6.b). The displacement of this node is calculated using:

$$
\left[u_{n_{R}+i-1}, u_{n_{R}+i-2}, \ldots, u_{i+1}, u_{i}\right]^{T}=\widetilde{K}_{R}^{-1} *\left[0,0, \ldots 0, R_{e}\right]^{T}
$$

Finally, the equivalent stiffness and the equivalent force representing the impact of the spans at the right side of the pole of interest are calculated as: 


$$
F_{R, E q}=R_{e} ; K_{R, E q}=\frac{R_{e}}{u_{i}}
$$

The same approach can be followed to derive the equivalent stiffness and wind force for the distribution line on the left side of the pole of interest. Subsequently, the equivalent stiffness and the equivalent force of the two sides can be added, since they work in parallel with respect to the pole of interest:

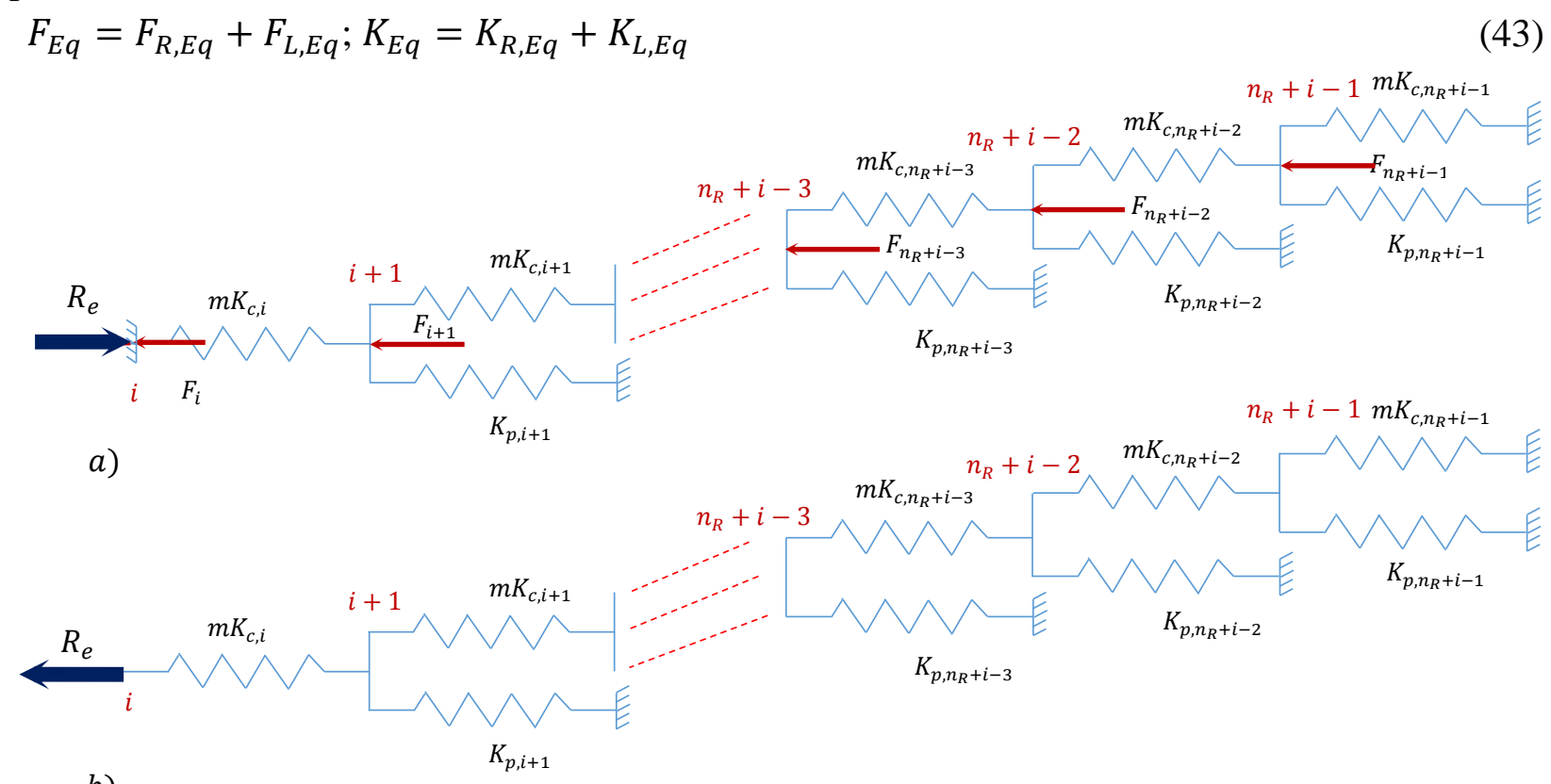

b)

Figure 6. Obtaining equivalent boundary conditions: a) fixing node $i$ and b) applying $-R_{e}$ to node $i$

\section{PROBABILITY OF FAILURE OF WOOD POLES IN OVERHEAD DISTRIBUTION LINES}

For any structure, failure to meet a particular performance level is an uncertain event. This uncertainty stems from the demand applied to the structure and the capacity of its resisting components. The probability of failure of a structure can be assessed by evaluating the uncertainty in the demand and capacity. In reliability assessment, the failure is defined using a limit state function represented by $G(X)$, where $G(X)<0$ represents the failure of the structure. Incorporating the law of total probability, the probability of failure of a system with limit state $G(X)$ is defined as

$$
P_{f}=P[G(X)<0]=\int_{y} P[G(X)<0 \mid I M=y] f_{I M}(y) d y
$$

where $I M$ represents the intensity measure, or a random variable that defines the intensity of the demand, $f_{I M}(y)$ is the probability density function of the intensity measure of the hazard, and $P[G(X)<0 \mid I M=y]$ is the fragility function that defines the conditional probability of failure given that $I M=y$.

The probability of failure of wood poles in overhead distribution lines under wind loadings can be obtained by using Eq. (44). Previously, Shafieezadeh et al. (2014) obtained the fragility functions for poles without considering the impact of adjacent spans on the pole of interest. In this 
study, the probability of failure of wood poles is assessed considering the impact of adjacent spans. Toward this goal, the limit state function describing the failure event of utility poles is defined as

$$
G(X)=R-S
$$

where $R$ is the moment capacity of the wood poles and $S$ is the wind-induced moment demand on the poles. The capacity and demand models for wood poles are presented in the following subsections.

\subsection{Capacity Model for Wood Poles}

A primary mode of failure in utility poles corresponds to the case of moment demands induced by wind loads exceeding the moment capacity of the poles (Shafieezadeh et al., 2014; Ryan et al., 2014; Salman and Li, 2015; and Salman et al., 2015). The cross section of the poles and correspondingly their moment capacity decrease with the height of the section in the poles. However, due to the contact with surrounding soil, the ground line has the highest rate of decay in the poles. In addition, it can be shown that the pole section with maximum moment demand is located very close to the soil surface. These conditions lead the pole section at the ground line to be vulnerable against failure under wind loadings. Therefore, the limit state function describing the failure of the poles is developed for the section at the ground line. Another possible failure mode is the failure of the foundation of utility poles; this type of failure is not considered in this study.

The modulus of rupture of wood poles at the ground line decreases as the size of the pole increases. Wolfe et al. (2001) proposed the following model for calculating the 5\% exclusion limit for modulus of rupture of wood poles based on regression analysis on experimental test data.

$$
\sigma_{R 5}=K_{S} K_{c} K_{h} K_{L} A C_{G L}^{B}
$$

where $C_{G L}$ is the circumference of the pole at the ground line, and $A$ and $B$ are regression parameters. For Southern Pine wood poles, these parameters were obtained as $3.482 \times 10^{7}$ and 0.320 , respectively which represent a $50 \%$ confidence in the lower $5 \%$ exclusion limit assuming a lognormal distribution for the strength of the poles. $K_{S}$ is the adjustment factor for the size effect which is equal to 1.1. $K_{c}$ is the adjustment factor for conditioning; it takes a value of 1 for air drying, 0.9 for kiln drying and Boulton drying and 0.85 for steaming. $K_{h}$ is the calibration factor for the historic precedent in conventional design which in this study is set as 1 . Finally, $K_{L}$ is the adjustment factor for the effect of load sharing (Wolfe et al., 2001). In this study, since load sharing between poles are considered through defining boundary conditions, this value is set as 1 . Equation (46) is an empirical relationship and the parameters $A$ and $B$ are found such that $\sigma_{R 5}$ is in $N / m^{2}$ when $C_{g l}$ is in $m$. Shafieezadeh et al. (2014) proposed a lognormal distribution for modulus of rupture of wood poles. In their model, the mean value of the modulus of rupture is derived as

$$
E\left(\Sigma_{R}\right)=E\left(\sigma_{R 5}\right) \times \exp \left[k_{0.05,0.5, \infty} \times \sqrt{\ln \left(\rho_{R}^{2}+1\right)}\right]
$$

where $\Sigma_{R}$ is a random variable representing the modulus of rupture of wood poles and $\rho_{R}$ is the coefficient of variation (COV) of the modulus of rupture of wood poles. The recommended value of COV for Southern Pines is 0.169 by ANSI 05.1 (2008). $k$ is the tolerance factor which can be derived from

$$
k_{\beta, \gamma, n}=\frac{1}{\sqrt{n}} \times t_{n-1 ; 1-\gamma}\left(z_{\beta} \sqrt{n}\right)
$$

where $t_{n-1 ; 1-\gamma}(\delta)$ denotes the $1-\gamma$ quantile of a non-central $t$ distribution with degrees of freedom of $n-1$ and the non-centrality parameter $\delta$, and $z_{\beta}$ denotes the $\beta$ quantile of standard 
normal distribution. Subsequently, the moment capacity of wood poles at the ground line can be determined as

$$
M_{G L}=\frac{\Sigma_{R} \cdot C_{G L}^{3}}{32 \pi^{2}}
$$

For a specific wood species, the moment capacity can be obtained from Eq. (49) when the ground line circumference is known. For reliability assessment of utility poles in the next sections, Monte Carlo simulations will be performed; for this purpose, samples of ground line moment capacity of wood poles need to be generated. ANSI O5.1 (2008) provides the minimum circumference at the tip of the poles for each class of pole and a minimum circumference at 6 feet from bottom for different classes and lengths of the poles. Using these information, the minimum ground line circumference for each class can be determined given the length of the pole. In this case, samples of ground line circumference for class $n$ wood poles can be generated assuming that the ground line circumference follows a uniform distribution where the lower and upper bounds of the uniform distribution are the ground line circumference for class $n$ and class $n-1$, respectively.

\subsection{Effect of In-Service Decay on Pole Capacity}

Wood poles may face decay of different levels of severity over their service life. The rate of decay in wood poles increases by warm temperatures, moisture, and oxygen from the atmosphere (Morrell, 1996). Due to the low rate of oxygen under the ground level, the segment of the pole which is buried under ground has a low rate of decay. However, the ground line has hold the highest rate of decay because it is exposed to oxygen from atmosphere, and the level of moisture transferred from soil is high compared to upper levels. Mechanistically, decay reduces the moment capacity of a section through reduction in the fiber strength and effective cross section area. A wood pole cross section is divided into two layers; the inner layer named heartwood and the outer layer called sapwood. The rate of decay depends on the thickness and durability of heartwood and sapwood (Shafieezadeh et al., 2014). Based on the species of the wood, the thickness and durability of each layer varies. For Southern Pine, which is used in this study, decay is external since the sapwood is thick. However, in some other species, the decay is mostly internal (Shafieezadeh et al., 2014). External decay reduces the diameter of wood poles, which results in a reduction in moment capacity of wood poles. It should also be noted that decay is a time-dependent phenomenon which is impacted by the species of wood, environmental conditions, such as moisture and temperature, and the treatment of wood. A time-dependent model for decay in wood poles is proposed by Shafieezadeh et al. (2014). This model provides time-dependent mean and variance of the capacity of wood poles in terms of the initial capacity. Based on this model, the expected value and variance of the capacity of wood poles are defined as

$$
\begin{aligned}
& E[R \mid T=t]=E\left[R_{0}\right]\left[1-\min \left(\max \left(a_{1} t-a_{2}, 0\right), 1\right) \times \min \left(\max \left(b^{\prime}{ }_{1} t^{b^{\prime}}{ }_{2}, 0\right), 1\right)\right] \\
& \operatorname{Var}[R \mid T=t]=\left(\operatorname{Var}\left[R_{0}\right]+E\left[R_{0}\right]^{2}\right)\left(1-\min \left(\max \left(b^{\prime}{ }_{1} t^{b^{\prime}}{ }_{2}, 0\right), 1\right)\right) \\
& +\left\{\left(\operatorname{Var}\left[R_{0}\right]+E\left[R_{0}\right]^{2}\right)\left[\operatorname{Var}[L \mid T]+\left(1-\min \left(\max \left(a_{1} t-a_{2}, 0\right), 1\right)\right)^{2}\right]\right\} \\
& \times\left(\min \left(\max \left(b^{\prime}{ }_{1} t^{b^{\prime}}{ }_{2}, 0\right), 1\right)\right)-E\left[R_{0}\right]^{2} \times[1 \\
& \left.-\min \left(\max \left(a_{1} t-a_{2}, 0\right), 1\right) \times \min \left(\max \left(b^{\prime}{ }_{1} t^{b^{\prime}{ }_{2}, 0}\right), 1\right)\right]^{2}
\end{aligned}
$$

where $R$ is the capacity of wood poles, $R_{0}$ is the capacity of the new poles, $t$ is the age of the wood pole in terms of years, and $\operatorname{Var}[L \mid T]$ is the time-dependent variance of the loss of the capacity of wood poles. According to Li et al. (2005), the variance of the loss of capacity of wood poles is set 
as 0.11. Parameters $a_{1}$ and $a_{2}$ define the percentage of strength loss for wood poles and based on a regression analysis performed by $\mathrm{Li}$ et al. (2005), they are found as 0.014418 and 0.10683 , respectively. Parameters $b^{\prime}{ }_{1}$ and $b^{\prime}{ }_{2}$ account for the percentage of decayed poles and according to the regression analysis performed by Shafieezadeh et al. (2014), they are found as 0.00013 and 1.846 , respectively.

\subsection{Stiffness Model for Wood Poles}

In this study, it is assumed that the uncertainty in the stiffness provided by a span in distribution lines stems from properties of wood poles and the stiffness of the other components of the distribution line is assumed deterministic. This is a reasonable assumption considering the high variation in material properties of wood compared to engineered components such as conductors. To obtain the probabilistic model for the stiffness of the poles, a regression analysis is performed to derive a relationship between their modulus of elasticity and modulus of rupture. Test data used for this regression analysis are obtained from experiments conducted by Anthony et al. (1992) on thirteen class five Southern Yellow Pine wood poles. Figure 7 shows the test data and the results of the regression analysis. Since the modulus of elasticity must be zero if the modulus of rupture is zero, the regression analysis is set to go through the origin. The analysis yields the following relationship for the elastic modulus, $E_{w}$ :

$$
E_{w}=206 \Sigma_{R} \times \varepsilon
$$

where $\Sigma_{R}$ is the random variable representing the modulus of rupture of wood poles and $\varepsilon$ is a random variable that accounts for the uncertainty in the regression model. The coefficient of determination, $R^{2}$, for this regression function is found equal to 0.5842 . As presented in section 3 , $\Sigma_{R}$ follows a lognormal distribution. In addition, $\varepsilon$ is assumed to follow a lognormal distribution with mean equal to 1.02 and standard deviation of 0.16 based on the test data and regression model. Knowing the distribution of $\Sigma_{R}$ and $\varepsilon$, samples of $E_{w}$ can be generated for Monte Carlo simulations.

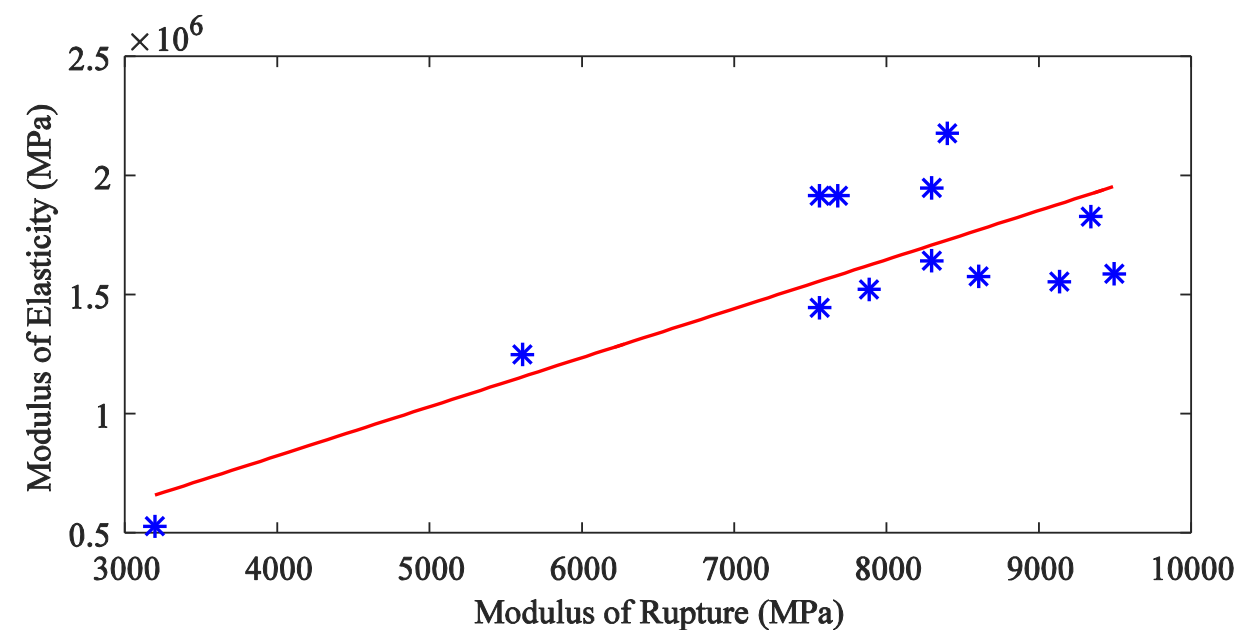

Figure 7. Regression analysis of thirteen class five southern yellow pine poles.

\subsection{Demand Model for Wood Poles}

Wind load on the poles depends on a number of factors such as the velocity and direction of wind, configuration and geometry of poles and conductors, roughness of the surface of poles, and number, diameter, and elevation of conductors in the lines. ANSI O5.1 (2008) classifies wood poles based on their geometric features to classes one through ten with classes one and ten having 
the largest and smallest circumferences, respectively. Shafieezadeh et al. (2014) observed that class three and five poles are among the most common classes of wood poles used in distribution lines. In this study, class five poles are considered to investigate the impact of adjacent spans on the wind performance of utility poles. The span length is another important geometric feature for the wind load on conductors and the stiffness of these components. In a study by Vickery et al. (1996), a lognormal distribution is used to define the span length for $11 \mathrm{kV}$ distribution lines. The mean and standard deviation of the lognormal distribution model are $43.9 \mathrm{~m}$ and $11.2 \mathrm{~m}$, respectively. The height of class five wood poles follows a lognormal distribution with mean and standard deviation of $11.4 \mathrm{~m}$ and $1 \mathrm{~m}$, respectively (Shafieezadeh et al., 2014). As stated previously in section 2.2, ASCE07 (2010) provides a set of models for wind loads on structures. Equation (3) defines the wind load per unit length for a non-building structure such as distribution lines. The mean value of the parameters involved in the wind force calculation was presented in section 2.2. While some of these parameters are defined by users and assumed to be deterministic, some others are considered probabilistic. Table 2 presents the probability distribution model and the coefficient of variation of the probabilistic parameters which are adopted from Ellingwood and Tekie (1999). Knowing the distribution and mean and COV of each probabilistic parameter, Monte Carlo simulations can be performed.

Table 2. Wind Load Statistics for ASCE07 (2010)

\begin{tabular}{ccc}
\hline Parameter & Distribution & COV \\
\hline$G$ & Normal & 0.11 \\
$C_{f}$ & Normal & 0.12 \\
$K_{z}$ & Normal & 0.16 \\
$K_{d}$ & Normal & 0.08 \\
\hline
\end{tabular}

\section{NUMERICAL STUDY}

\subsection{Validation of the Approximate Shape of Conductors under Lateral Static Gust Wind and Gravity Loadings}

In section 2.3.2, it was proposed that if the sag is negligible compared to the span length, the exact solution for the deformed shape of conductors under static gust wind and gravity loadings can be approximated by a parabolic function. In this section, in order to assess the accuracy of the approximate model, its solutions are compared with the exact solutions and numerical results of Finite Element analysis of conductors in OpenSEES (McKenna, 2000). For this purpose, a single conductor pinned at both ends is considered under lateral static gust wind and gravity loadings. The boundary conditions (i.e. the location of the pins) are assumed to be at $(0,1,0.15) \mathrm{m}$ and $(L, 0.5,0.1) \mathrm{m}$, where $L$ is the span length. The conductor used in this study is an Aluminum Conductor Steel Reinforced (ACSR) type with $26 \mathrm{~mm}$ equivalent diameter, 73.2 MPa modulus of elasticity, and $1.81 \mathrm{~kg} / \mathrm{m}$ mass. The ultimate tensile capacity of the conductor is $147 \mathrm{kN}$. The horizontal component of initial tension in the conductor is considered to be $30 \%$ of its tensile capacity. A lateral static gust wind speed of $33.3 \mathrm{~m} / \mathrm{s}(75 \mathrm{mph})$ is applied to the conductor. An elastic perfectly plastic gap material with tension-only behavior is also used to perform numerical Finite Element analysis of conductors in OpenSEES. The exact deformed shape of the conductor from Eq. (26) and the approximate deformed shape from Eqs. (29) and (30) are compared with the results from the Finite Element analysis for different span lengths and the results are presented in Figure 8. It is seen that all three solutions have a very good agreement; in particular, the difference between the solutions of the exact and approximate model is less than $0.1 \%$. The maximum 
difference in the deformed shape of the conductor derived from exact and approximate model is also assessed for a broad range of wind speeds $(15 \mathrm{~m} / \mathrm{s}$ to $55 \mathrm{~m} / \mathrm{s}$ ) and span lengths ( $30 \mathrm{~m}$ to 120 $\mathrm{m})$. These results are presented in Figure 9 for the deformed shape of conductor in $y$ and $z$ directions. The maximum difference is $0.2 \%$ which indicates the high accuracy of the approximate model.

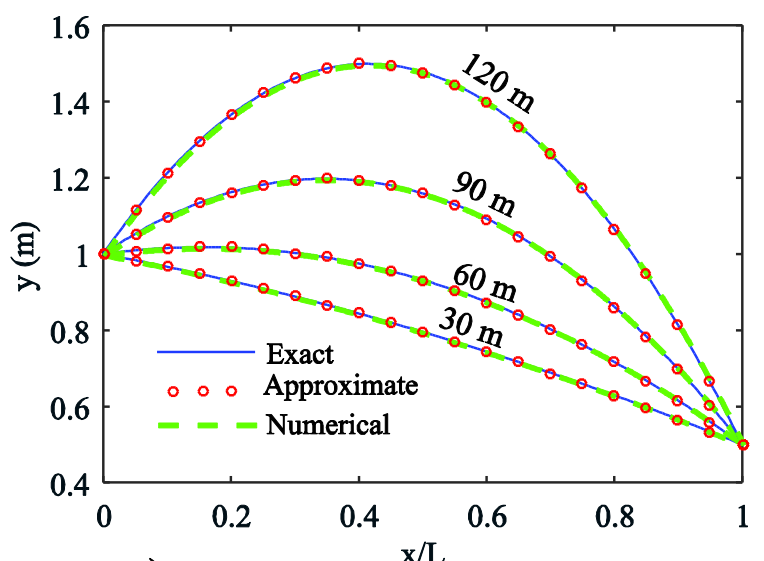

a)

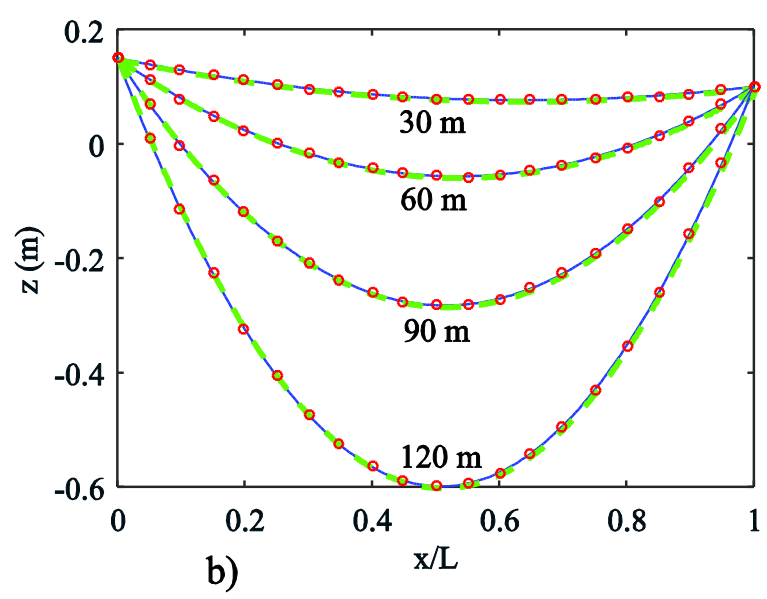

Figure 8. Exact, approximate and FE derived shape of conductor for different span lengths (L) in (a) y-direction and (b) z-direction for a wind speed of $33.3 \mathrm{~m} / \mathrm{s}(75 \mathrm{mph})$.

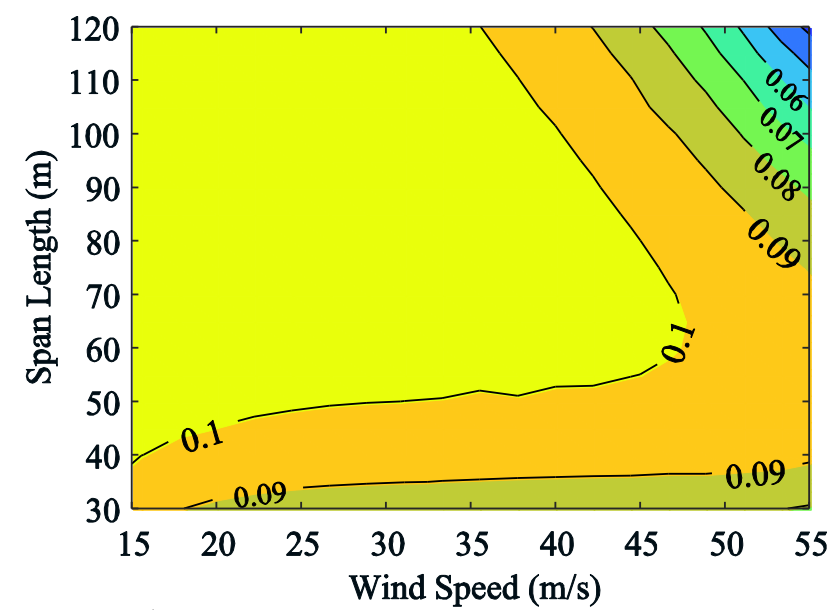

a)

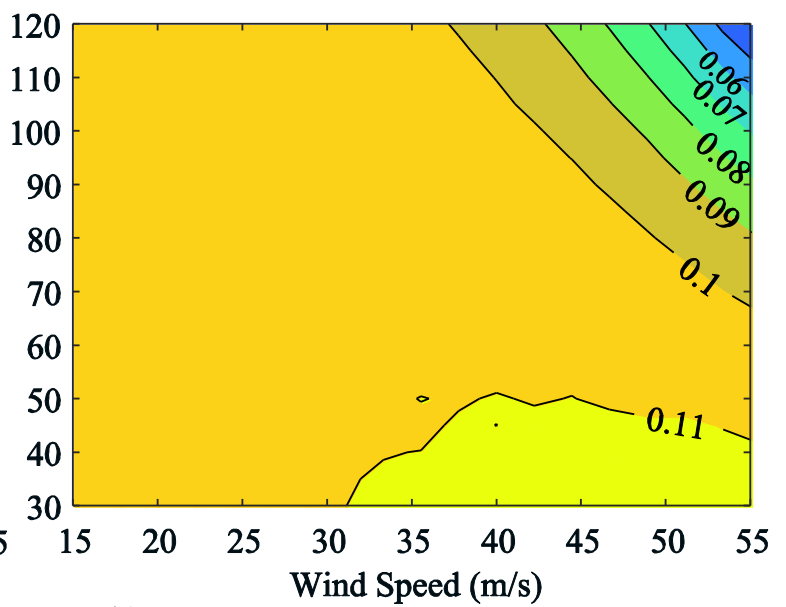

b)

Figure 9. Difference (\%) of approximate vs numerical solutions for the deformed shape of cables for (a) y-direction and b) z-direction.

\subsection{Effects of Age Distribution and Number of Spans on the Equivalent Boundary Conditions}

The simplest case for a distribution line is to assume that all spans are exactly identical in terms of stiffness and the wind-induced forces. Therefore, under static gust wind loads, all the poles experience identical displacements. In this case, the impact of the stiffness of the conductors can be neglected because each two adjacent poles have the same displacement and therefore, there is no relative displacement to impose the impact of the stiffness of the conductors. However, in reality especially in the case of old poles which have been exposed to decay and deterioration, the 
situation is different. Often, poles are inspected every few years and a percentage of them that are decayed are replaced with new poles. In addition, following hurricanes and strong winds, utility poles that are collapsed or damaged beyond repair are replaced with new poles. Therefore, there is a variation in the age of the poles in distribution lines. Due to this variation in the age of the poles and the corresponding decay associated with old poles, there is a variation in the stiffness and strength of wood poles. Therefore, the situation in which a pole with high stiffness is located next to a pole with low stiffness is highly probable. Subsequently, for obtaining the response or assessing the performance of wood poles, especially in the case of old distribution lines, the impact of neighboring spans on the pole of interest must be investigated.

Another influential factor for the equivalent boundary effects for the pole of interest is the number of spans on each side of the pole. As mentioned before, the first few spans next to the pole of interest may have a considerable impact on the response of the pole. To investigate this effect along with those from the age of the poles, the convergence of the ground line moment in the pole of interest with respect to the number of spans is examined based on Eqs. (34) - (43) for an overhead distribution line shown in Figure 10.a. In this example, a deterministic study is performed and a probabilistic study is provided in the next subsection. For the deterministic study, all of the probabilistic variables are set to their expected values. The assumed distribution line in this example consists of two lines of conductors similar to the distribution line presented in Figure 1 made of ACSR conductors with $14.3 \mathrm{~mm}$ diameter which based on US naming system for ACSR conductors represents Penguin conductor. Class five poles are considered with $15.24 \mathrm{~m} \mathrm{(50} \mathrm{ft)}$ height, $0.16 \mathrm{~m}(6.1 \mathrm{inch})$ diameter at the top and $0.28 \mathrm{~m}(10.8 \mathrm{inch})$ diameter at $1.83 \mathrm{~m}(6 \mathrm{ft})$ from the bottom (ANSI 05.1, 2008). The ground line distance from the bottom is assumed to be equal

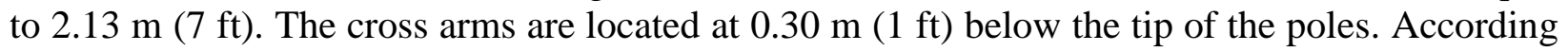
to Vickery et al. (1996), the span length for $11 \mathrm{kV}$ distribution lines follows a lognormal distribution with a mean and standard deviation of $43.9 \mathrm{~m}$ and $11 \mathrm{~m}$, respectively. Three span lengths corresponding to $10 \%$, mean, and $90 \%$ of the CDF of this lognormal distribution are used in this study. The span lengths are found to be $30.8 \mathrm{~m}, 43.9 \mathrm{~m}$ and $58.7 \mathrm{~m}$, respectively. A static gust wind speed of $30 \mathrm{~m} / \mathrm{s}(67.1 \mathrm{mph})$ is applied to the distribution line

The relative stiffness of adjacent spans defines the impact of neighboring spans on the pole of interest. Since the modulus of elasticity and rupture are positively correlated as shown in Figure 7 , it is expected that the stiffness of poles decreases with their age. Consequently, poles of different age are likely to have different stiffnesses (Figure 10.b). In this example, pole 6 in Figure 10 is chosen as the pole of interest. The eleven poles shown in Figure 10 are part of a long distribution line with all the poles that are not shown in the figure are $t_{a}$ years old. The modulus of elasticity of the poles is determined based on Eq. (52) with the mean value for the modulus of rupture determined through Eqs. (47) and (50). In Figure 11, the results of the numerical study for the wind-induced ground line moment of the pole of interest versus the number of spans are presented. The results are presented for the case with no boundary effects considered (denoted by NBC in Fig. 11) and the case where boundary effects are accounted for (denoted by BC in Fig. 11). Considering that the span lengths, number of conductors, and wood poles are all the same, Figure 11.a shows clearly that if poles have the same age, the stiffnesses of all the spans will be the same. Therefore, adjacent spans do not impact the pole of interest. The ground line moment of the pole of interest in the case of no boundary effects (in Figure 11.a denoted by NBC) is equal to the case in which the ground line moment of the pole of interest converges after considering a few spans (number of spans larger than 4 in Figure 11. a denoted by BC). On the other hand, when poles have different ages, the stiffness of the spans are different. Therefore, adjacent spans impose 
considerable impact on the pole of interest. In Figure 11.b, the ground line moment of pole 6, in the case of no boundaries, is different from the case that the ground line moment converges after considering a few spans. Considering the results of this numerical study, it can be concluded that if there is considerable variation in the stiffness of adjacent spans, boundary conditions must be considered for analyzing the performance of the pole of interest.

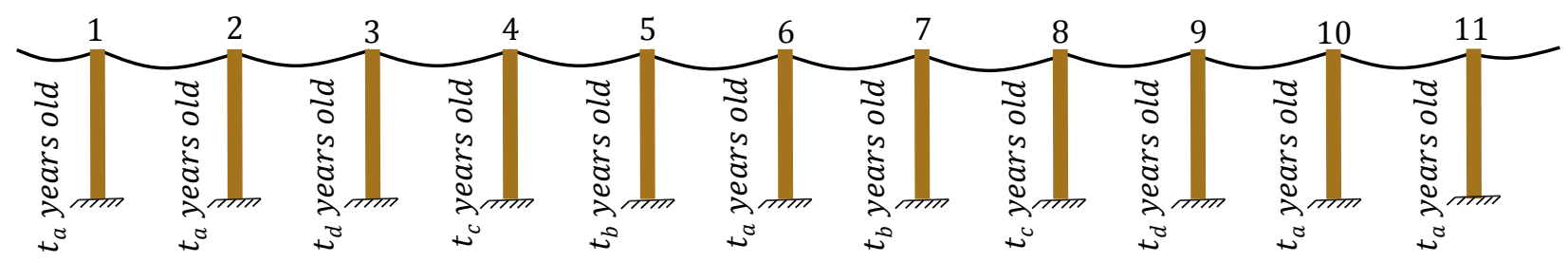

a)

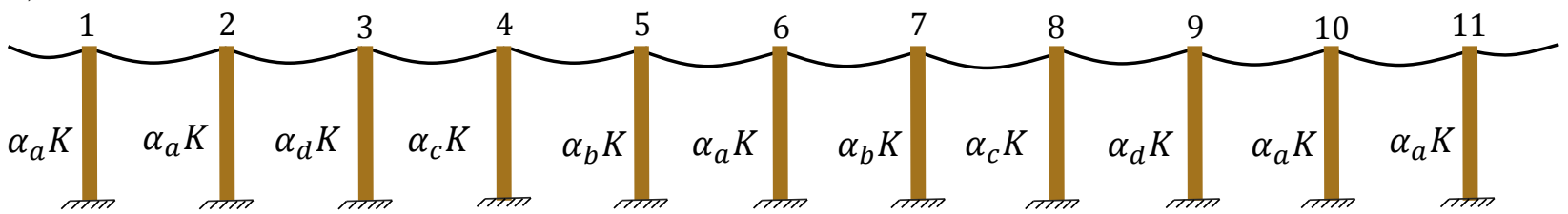

b)

Figure 10. Assumed distribution line for the numerical study: (a) age of poles and (b) stiffness of poles.

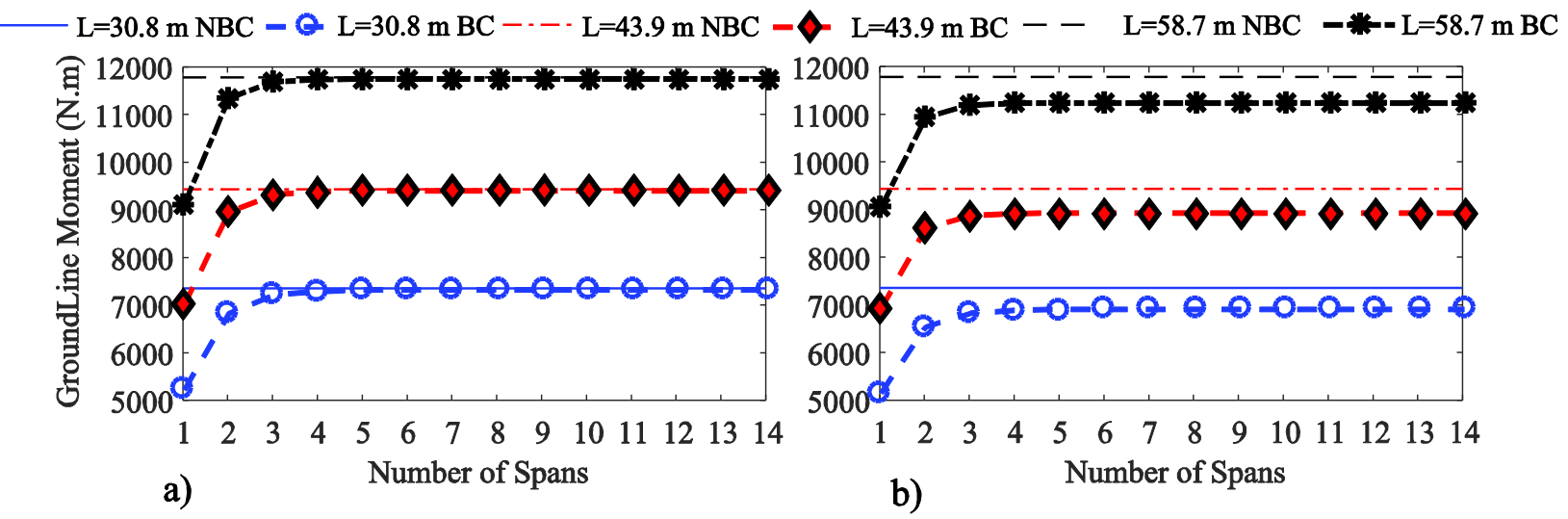

Figure 11. Ground line moment of pole 6 vs. number of spans for gust wind speed of $30 \mathrm{~m} / \mathrm{s}(67.1$ $\mathrm{mph}$ ) when boundary conditions are considered (denoted by BC) and when boundary conditions are not considered (denoted by NBC) for (a) $t_{\mathrm{a}}=t_{\mathrm{b}}=t_{\mathrm{c}}=t_{\mathrm{d}}=60$ years and (b) $t_{\mathrm{a}}=60$ years, $t_{\mathrm{b}}=0$ years, $t_{\mathrm{c}}=20$ years and $t_{\mathrm{d}}=40$ years.

\subsection{Probability of Failure of Wood Poles in an Overhead Distribution Line Considering Boundary Conditions}

In this section, the impact of boundary conditions on the probability of failure of a set of poles with different ages in a distribution line is investigated. For this purpose, the time-dependent decay model for the capacity of wood poles and the probabilistic wind model presented in section 3 are used to perform a Monte Carlo simulation to obtain the probability of failure of the utility poles. In order to consider effects of the boundary conditions, a probabilistic model for the stiffness of spans must also be included. The geometry and modulus of elasticity of a wood pole defines its stiffness as presented in Eqs. (1) and (2). The modulus of elasticity of poles is derived using the 
probabilistic model for the modulus of rupture through Eq. (52) considering that the age of the poles is known.

In order to investigate the impact of boundaries on the probability of failure of wood poles, a distribution line with class 5 poles and four lines of conductors are considered (Figure 10.a). In this example, the poles are assumed to be $t$ years old, except two of them, which are new poles (poles number 4 and 8 in Figure 10.a) that recently replaced old poles. The height of the poles are assumed to be $11.3 \mathrm{~m}$ and the conductors are ACSR with $14.3 \mathrm{~mm}$ diameter. Based on the US naming system for conductors, the conductor represents a Penguin conductor. The span length is assumed to be $43.9 \mathrm{~m}$. A Monte Carlo simulation is performed using 20000 samples generated by Latin Hypercube sampling method to obtain the probability of failure of the wood poles. In order to find the probability of failure, the wind-induced moment is compared with the moment capacity of wood poles at the ground line. The impact of adjacent spans on the probability of failure of each pole is considered through equivalent boundary conditions presented in this paper. In Figure 12, the probability of failure of wood poles for different wind speeds is shown. It is observed that for different wind speeds when the boundaries are considered, the probability of failure of new poles increases (Pole 4 and 8 ) and the probability of failure of old poles decreases. For example, for the wind velocity of $53.6 \mathrm{~m} / \mathrm{s}(120 \mathrm{mph})$, the probability of failure of poles 4 and 5 is 0.03 and 0.32 , respectively when no boundary effects are considered. These failure probabilities change to 0.12 and 0.27 when boundary effects are taken into account indicating a 300\% increase and $16 \%$ decrease in the probability of failure of poles 4 and 5, respectively. Since new poles have higher stiffness compared to old poles, when the boundaries are considered, they carry a larger force. This results in an increase in the moment demand in new poles and decrease in the demand in old poles. It is also observed that the poles which are far from poles 4 and 8 are less impacted by the boundary conditions, but the impact on the poles next to poles 4 and 8 is considerable. It again verifies that the impact of the first few poles next to the span of interest is considerable, while the poles that are far from the pole of interest have negligible impact.

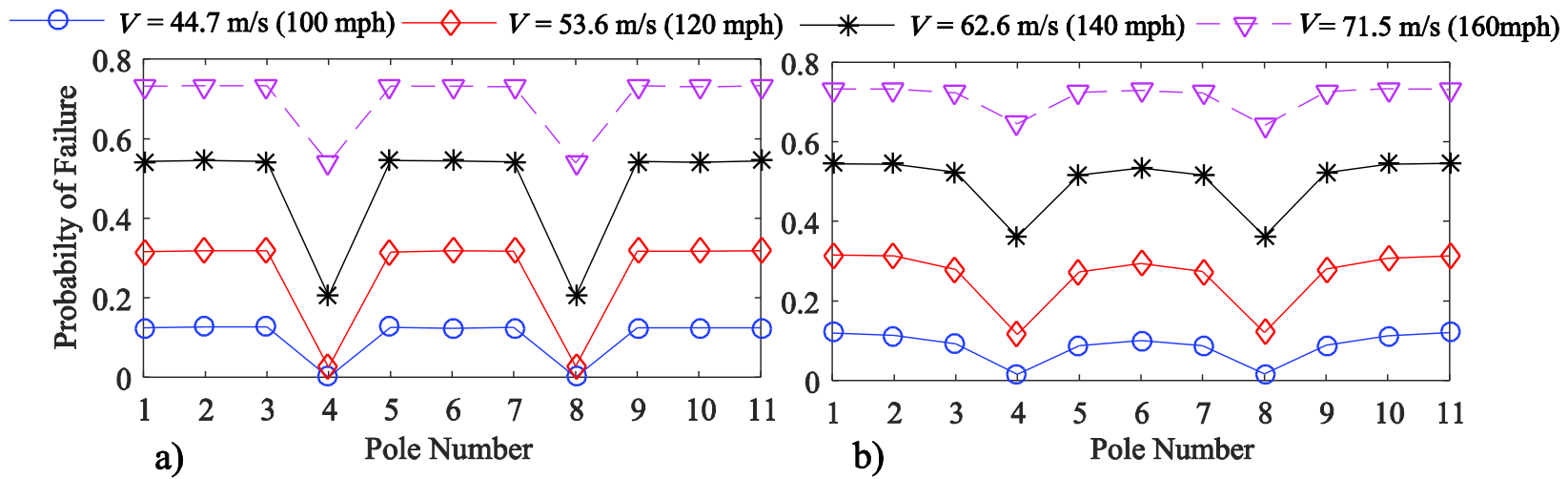

Figure 12. Probability of failure of poles in the assumed distribution line of Figure 10 for $t_{a}=t_{b}=t_{d}=60$ years and $t_{c}=0$ years for the case of (a) without boundary conditions and (b) with boundary conditions.

The probability of failure of wood poles for the wind velocity of $71.5 \mathrm{~m} / \mathrm{s}(160 \mathrm{mph}), t_{c}=0$, and different values of $t$ where $t_{a}=t_{b}=t_{d}=t$ is presented in Figure 13. Similar to results shown in Figure 12, considering boundary conditions appear to increase the probability of failure of new poles since they have higher stiffness and decrease the probability of failure of older poles. Furthermore, when boundary effects are not considered (Figure 13.a), the probability of failure of 
new poles remains the same irrespective of the age of the poles next to the new poles, but when the boundaries are considered (Figure 13.b), the probability of failure of new poles is significantly impacted by the age of the rest of the system. For $t=70$ years and boundary effects, the probability of failure of the new poles (poles 4 and 8 ) is 0.55 ; considering boundary effects increases this value by $27 \%$ to 0.70 .

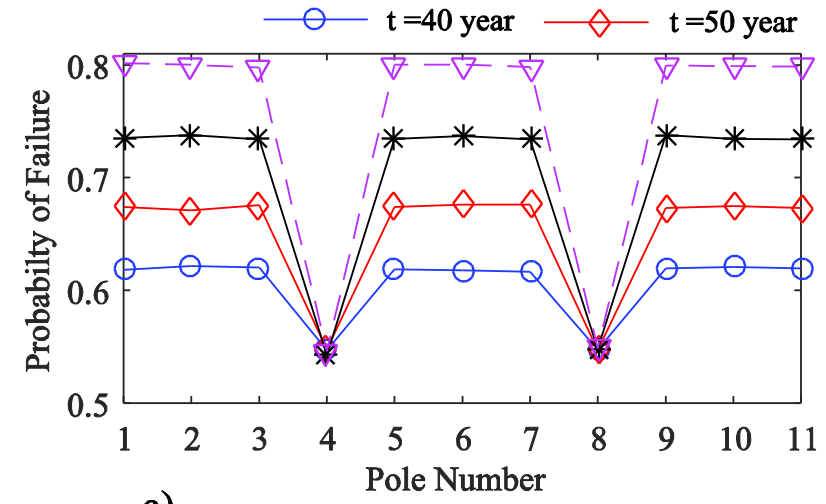

a)

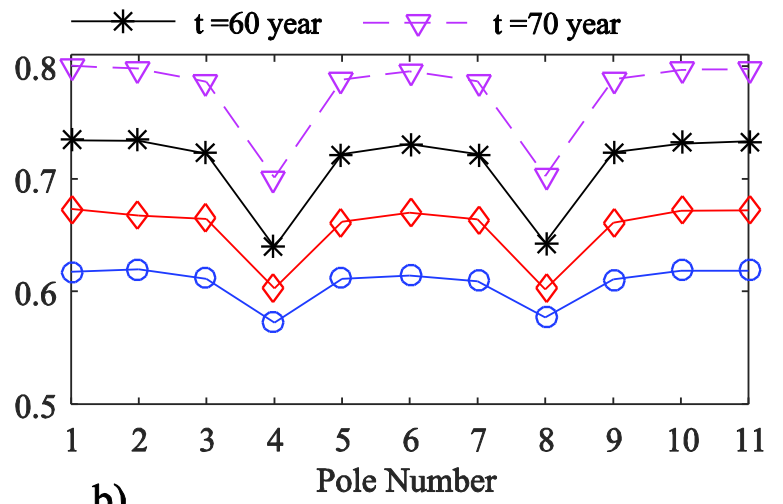

b)

Figure 13. Probability of failure of poles in the assumed distribution line of Figure 10 for $t_{a}=t_{b}=t_{d}=t$ years, $t_{c}=0$ years and wind speed equal to $71.5 \mathrm{~m} / \mathrm{s}$ (160 mph) for the case (a) without boundary conditions and (b) with boundary conditions.

These results indicate that in case adjacent poles in a distribution line do not have the same age, their stiffness is likely to be different; depending on the extent of this difference, boundary effects may considerably impact the performance of the poles. Variation in the stiffness and force of adjacent spans can be caused by differences in the age and geometry of poles, span length, number and diameter of conductors, and wind speed and its direction. If there is a large difference in any of these variables among adjacent poles, boundary effects must be included in the performance assessment of wood poles. On the contrary, if there is no or negligible variation in the stiffness and force of adjacent poles, boundary effects can be neglected.

\section{SUMMARY AND CONCLUSIONS}

In the performance assessment of overhead distribution lines, it is not feasible to model the entire system because distribution lines are very long and consist of a large number of spans. In order to overcome this issue, the segment of interest in the distribution line can be modeled and the impact of the rest of the system can be considered through equivalent boundary conditions. In this paper, a method is proposed to define the equivalent boundary conditions to account for the effects of neighboring spans on the pole or segment of interest. The boundary model consists of equivalent springs and forces for each component of the distribution line. The exact solution for the deformed shape of a segment of conductors under three-dimensional boundary conditions and combined lateral static gust wind and gravity loadings is derived. In order to obtain the equivalent stiffness of conductors, an approximate model for the shape of conductors is developed. It is shown that this solution accurately approximates the exact solution and that of Finite Element models. For a broad range of wind speeds and span lengths, solutions of all three models are found to be very close with a difference of less than $0.2 \%$. Using the approximate solution for the three-dimensional deformed shape of conductors, an equivalent stiffness and force for conductors when subjected to lateral gravity and gust wind loadings are derived. Subsequently, a set of stiffness matrices and force vectors are constructed to model the response of segments of distribution lines under lateral 
gust wind loadings. These models are utilized to obtain the equivalent stiffness and force that represent the boundary effects of adjacent spans on the response of the pole of interest in distribution lines. The proposed models provide an accurate yet practical method to properly account for boundary effects and investigate the performance of distribution lines against wind hazards.

Deterministic and probabilistic studies are performed on a set of wood poles in an overhead distribution line. The impact of boundary conditions is investigated for the ground line moment demand and the probability of failure of wood poles. Results reveal that adjacent spans may have considerable impact on the performance of the pole of interest. This impact becomes more significant when the extent of the difference in the stiffness and wind-induced forces of the spans next to the pole of interest is not negligible. In a distribution line that consists of poles with different ages, considering boundary conditions increases the probability of failure of newer poles and decreases the probability of failure of older poles. For a case study line, boundary effects lead to an increase of $300 \%$ and decrease of $16 \%$ in the probability of failure of a new and an old pole, respectively. This is due to the fact that new poles have higher stiffness compared to old poles, and therefore they carry higher levels of load in the line of poles. The impact of boundaries on the probability of failure of wood poles increases as the difference in the age of the neighboring poles increases. Other factors that may result in boundaries having high impact on the performance of the poles include differences in the geometry of the poles, span length, number and diameter of conductors, and wind speed and direction between adjacent spans. The developed boundary models release a simplifying assumption in current reliability studies of distribution lines to enhance the accuracy of wind performance assessments of utility poles. These models can be used in risk and life-cycle cost assessment frameworks to assist in decision making with regard to pole inspection and replacement.

\section{ACKNOWLEDGEMENT}

This paper is based upon work supported by the National Science Foundation under Grant No. CMMI-1333943 and 1334733. This support is greatly appreciated.

\section{REFERENCES}

ANSI O5.1, Specifications and Dimensions for Wood Poles, Amer. Nat. Std. Inst. (ANSI), New York, 2008.

Anthony, R. W., J. Bodig, G. E. Phillips, and R. T. Brooks. "Longitudinal nondestructive evaluation of new utility wood poles". No. EPRI-TR-100864-Vol. 1. Electric Power Research Inst., Palo Alto, CA (United States); Engineering Data Management, Inc., Fort Collins, CO (United States), 1992.

Blake, E. S., T. B. Kimberlain, R. J. Berg, J. P. Cangialosi, and J. L. Beven II. "Tropical cyclone report: Hurricane sandy." National Hurricane Center 12: 1-10, 2013.

Brown, R. E., in IEEE GM 2006 Working Group on System Design, Jun. 21, 2006. [Online]. Available: http://grouper.ieee.org/groups/td/dist/sd/doc/2006-06-Hurricane-Wilma.pdf

Davidson, R. A., H. Liu, I. K. Sarpong, P. Sparks, and D. V. Rosowsky. "Electric power distribution system performance in Carolina hurricanes." Natural Hazards Review 4, no. 1 : 36-45, 2003.

Ellingwood, B. R., and P. B. Tekie. "Wind load statistics for probability-based structural design." Journal of Structural Engineering 125, no. 4: 453-463, 1999.

Johnson, B., "Utility storm restoration response." Edison Electric Institute, Washington DC., 2004. 
Larsen, T., Porter, K., Zadeh, M., Van Anne, C., and Scawthorn, C., "Impact of Hurricane Andrew on performance, interaction, and recovery of lifelines", EQE International, San Francisco, 1996.

Li, Y., S. Yeddanapudi, J. D. McCalley, A. A. Chowdhury, and M. Moorehead. "Degradation-path model for wood pole asset management." In Power Symposium, Proceedings of the 37th Annual North American, pp. 275-280. IEEE, 2005.

Minimum Design Loads for Buildings and Other Structures Amer. Soc. Civil Eng. (ASCE). Reston, VA, USA, 2010, ASCE 07-10.

Morrell, J. J., "Wood Pole Maintenance Manual: 1996 Edition”. Forest Research Laboratory, Oregon State University, 1996.

Onyewuchi, U. P., A. Shafieezadeh, M. M. Begovic, and R. DesRoches. "A Probabilistic Framework for Prioritizing Wood Pole Inspections Given Pole Geospatial Data." Smart Grid, IEEE Transactions on 6, no. 2: 973-979, 2015.

Ryan, P. C., M. G. Stewart, N. Spencer, and Y. Li. "Reliability assessment of power pole infrastructure incorporating deterioration and network maintenance." Reliability Engineering \& System Safety 132: 261-273, 2014.

Salman, A. M., and Y. Li. "Age-dependent fragility and life-cycle cost analysis of wood and steel power distribution poles subjected to hurricanes." Structure and Infrastructure Engineering: $1-14,2015$.

Salman, A. M., Y. Li, and M. G. Stewart. "Evaluating system reliability and targeted hardening strategies of power distribution systems subjected to hurricanes." Reliability Engineering \& System Safety 144: 319-333, 2015.

Shafieezadeh, A., U. P. Onyewuchi, M. M. Begovic, and R. DesRoches. "Age-dependent fragility models of utility wood poles in power distribution networks against extreme wind hazards." Power Delivery, IEEE Transactions on 29, no. 1: 131-139, 2014.

Vickery, P. J., L. A. Twisdale, P. Montpellier, and A. C. Steckley. "Hurricane vulnerability and risk analysis of the VINLEC transmission and distribution system", Applied Research Associates; OAS. Caribbean Disaster Mitigation Project, 1996.

Wolfe, R. W., J. Bodig, and P. Lebow. "Derivation of nominal strength for wood utility poles.", U.S. Dept. Agriculture, Forest Service, Forest Products Lab., Madison, WI, USA, Rep. FPLGTR-128, 2001. 